\title{
Suizidraten im Zeichen der Sonne
}

Fragestellung: Beeinflusst die Sonnenscheindauer die Suizidhäufigkeit unabhängig von jahreszeitlichen Schwankungen?

Hintergrund: Seit etwa 200 Jahren wurde der Einfluss von jahreszeitlichen Schwankungen auf die Suizidraten untersucht. Es ist bekannt, dass suizidales Verhalten durch die Dauer des Sonnenscheins beeinflusst wird und jahreszeitlichen Mustern folgt; dies lässt sich durch den Einfluss von Licht auf das serotonerge System erklären. Jedoch bringen Jahreszeiten nicht nur Veränderungen meteorologischer Faktoren mit sich, sondern auch Veränderungen sozialer Variablen wie Feiertage, Arbeitslosigkeit etc. Daher stellt sich die Frage, inwieweit Sonnenschein die Suizidraten direkt beeinflusst.

Patienten und Methodik: Es wurden alle Daten offiziell bestätigter Suizide in Österreich zwischen dem 1. Januar 1970 und dem 6. Mai 2010 analysiert $(n=69.462)$. Für denselben Zeitraum wurde aus den Daten von 86 repräsentativen meteorologischen Stationen die mittlere tägliche Sonnenscheindauer (in Stunden) berechnet. Um saisonal bedingte Variationen der Sonnenstunden und der Suizidhäufigkeit zu eliminieren, wurde ein Differenzverfahren zwischen den Werten der Zeitreihen für Suizide und Sonnenstunden ange-

Vyssoki B, Kapusta ND, Praschak-Rieder $\mathrm{N}$ et al. Direct effect of sunshine on suicide. JAMA Psychiatry 2014; 71: $1231-7$ wandt. Anschließend wurde jeweils die Zahl der sich an einem bestimmten Tag ereigneten Suizide mit der Anzahl der Sonnenstunden an die- sem Tag oder für einen bestimmten Zeitraum von Tagen vorher korreliert.

Ergebnisse: Im Studienzeitraum ereigneten sich 69.462 Suizide. $28,5 \%$ der Suizidopfer waren Frauen und in 73,5\% wurde eine harte Suizidmethode gewählt. Die täglichen Sonnenstunden korrelierten eng mit der Zahl der Suizide $\left(r=0,4870 ; \mathrm{p}<10^{-9}\right)$. Es wurde eine positive Korrelation zwischen der Zahl der Suizide und der Zahl der Sonnenscheinstunden in den letzten zehn Tagen vor dem Suizid gefunden $\left(\mathrm{r}_{\text {maximum }}=0,0370 ; \mathrm{p}<10^{-5}\right)$. Eine negative Korrelation zwischen der Zahl der Suizide und den täglichen Sonnenscheinstunden bestand für den Zeitraum von 14 bis 60 Tagen vor dem Suizid $\left(r_{\text {minimum }}=-0,0383 ; p<10^{-5}\right)$. Diese Effekte wurden sowohl für die Gesamtgruppe als auch für die Gruppe der Suizidopfer mit harten Methoden gefunden. Bei weiblichen Suizidenten waren die positiven Korrelationen zwischen Zahl der Suizide und Sonnenscheindauer für die Periode der letzten zehn Tage vor dem Suizid $\left(\mathrm{r}_{\text {maximum }}=0,0388 ; \mathrm{p}<10^{-}\right.$ $\left.{ }^{5}\right)$ am höchsten; hingegen korrelierte bei den männlichen Suizidopfern die Zahl der Suizide mit der Sonnenscheindauer in der Periode vom 14. bis zum 60. Tag für den Suizid am stärksten (negativ; $r_{\text {minimum }}=-0,0346 ; p<0,001$ ).

Schlussfolgerungen: Die tägliche Sonnenstundenzahl korrelierte signifikant mit der Suizidhäufigkeit, unabhängig von der Jahreszeit, jedoch waren die Effektstärken niedrig. Abhängig von der Zeitperiode vor dem Suizid wirkte die Sonnenscheindauer suizidfördernd oder suizidprotektiv.

\section{- Kommentar von Barbara Schneider, Köln}

\section{Ein geringer, aber periodenabhängiger Effekt}

Es handelt sich um die erste Studie, in der aggregierte Daten eines Landes über einen langen Zeitraum von 40 Jahren mittels eines Differenzverfahrens von Zeitreihenanalysen zur Eliminierung des Einflusses saisonaler Varianz analysiert wurden.

Die Dauer des Sonnenscheins in den letzten zehn Tagen korrelierte positiv mit der Zahl der Suizide; dies galt auch für die Untergruppe der Frauen und für harte Suizidmethoden. Für den Zeitraum zwischen dem 14. bis zum 60. Tag vor dem Suizid korrelierte die Sonnenscheindauer negativ mit der Suizidhäufigkeit; dies zeigte sich auch in der Untergruppe, die harte Suizidmethoden anwendete. Die Sonnenscheindauer hatte somit einen suizidfördernden, aber auch einen suizidprotektiven Effekt, abhängig von der jeweiligen Zeitperiode vor dem Suizid.

Zum suizidfördernden Effekt von Sonnenscheindauer liegt inzwischen mehr als eine methodisch hochwertige, nichtexperimentelle Studie (Evidenzgrad IV) vor. Die Ergebnisse unterstreichen die Bedeutung des Einflusses von Sonnenlicht auf Suzidalität. Allerdings kann der Einfluss anderer Faktoren, wie klimatischer, sozialer oder genetischer Faktoren, mit diesem Studiendesign nicht untersucht werden. Klinisch Tätigen sollte der
Einfluss von Sonnenlicht auf Suizidalität bei der Beurteilung des Suizidrisikos bewusst sein, insbesondere der kurzfristige Suizidtriggernde Effekt von Sonnenlicht. Es gibt noch viele offene Fragen, zum Beispiel, ob die suizidfördernden und -protektiven Effekte von Sonnenlicht bei bestimmten psychischen Erkrankungen oder in Abhängigkeit von weiteren externen und internen, insbesondere biologischen Faktoren, besonders ausgeprägt sind und ob andere Variablen den Einfluss von Sonnenlicht moderieren können.

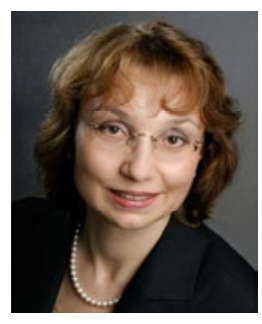

Prof. Dr. med. Barbara Schneider, M. Sc., Köln

LVR-Klinik Köln, Abteilung für Abhängigkeitserkrankungen und Allgemeinpsychiatrie E-Mail:B.Schneider@lvr.de 